\title{
On the Smarandache-Pascal derived sequences of generalized Tribonacci numbers
}

\author{
Zhengang $\mathrm{Wu}^{1 *}$, Jianghua $\mathrm{Li}^{2}$ and Han Zhang
}

${ }^{*}$ Correspondence: sky.wzgfff@163.com

'Department of Mathematics, Northwest University, Xi'an, Shaanxi, P.R. China

Full list of author information is available at the end of the article

\begin{abstract}
For any sequence recurrence formula, the Smarandache-Pascal derived sequence $\left\{T_{n}\right\}$ of $\left\{b_{n}\right\}$ is defined by $T_{n+1}=\sum_{k=0}^{n}\left(\begin{array}{l}n \\ k\end{array}\right) \cdot b_{k+1}$ for all $n \geq 2$, where $\left(\begin{array}{l}n \\ k\end{array}\right)=\frac{n !}{k !(n-k) !}$ denotes the combination number. The recurrence formula of $\left\{T_{n}\right\}$ is obtained by the properties of the third-order linear recurrence sequence.
\end{abstract}

Keywords: Smarandache-Pascal derived sequence; Tribonacci numbers; combination number; elementary method

\section{Introduction}

For any sequence $\left\{b_{n}\right\}$, a new sequence $\left\{T_{n}\right\}$ is defined by the following method: $T_{1}=b_{1}$, $T_{2}=b_{1}+b_{2}, T_{3}=b_{1}+2 b_{2}+b_{3}$, generally, $T_{n+1}=\sum_{k=0}^{n}\left(\begin{array}{l}n \\ k\end{array}\right) \cdot b_{k+1}$ for all $n \geq 2$, where $\left(\begin{array}{l}n \\ k\end{array}\right)=\frac{n !}{k !(n-k) !}$ is the combination number. This sequence is called the Smarandache-Pascal derived sequence of $\left\{b_{n}\right\}$. It was introduced by professor Smarandache in [1] and studied by some authors. For example, Murthy and Ashbacher [2] proposed a series of conjectures related to Fibonacci numbers and the Smarandache-Pascal derived sequence, one of them is as follows.

Conjecture Let $\left\{b_{n}\right\}=\left\{F_{8 n+1}\right\}=\left\{F_{1}, F_{9}, F_{17}, F_{25}, \ldots\right\},\left\{T_{n}\right\}$ be the Smarandache-Pascal derived sequence of $\left\{b_{n}\right\}$, then we have the recurrence formula

$$
T_{n+1}=49 \cdot\left(T_{n}-T_{n-1}\right), \quad n \geq 2 .
$$

Li and Han [3] studied these problems and proved a generalized conclusion as follows.

Proposition Let $\left\{X_{n}\right\}$ be a second-order linear recurrence sequence with $X_{0}=u, X_{1}=v$, $X_{n+1}=a X_{n}+b X_{n-1}$ for all $n \geq 1$, where $a^{2}+4 b>0$. For any positive integer $d \geq 2$, we define the Smarandache-Pascal derived sequence of $\left\{X_{d n+1}\right\}$ as

$$
T_{n+1}=\sum_{k=0}^{n}\left(\begin{array}{l}
n \\
k
\end{array}\right) \cdot X_{d k+1} .
$$

Then we have the recurrence formula

$$
T_{n+1}=\left(2+A_{d}+b \cdot A_{d-2}\right) \cdot T_{n}-\left(1+A_{d}+b \cdot A_{d-2}+(-b)^{d}\right) \cdot T_{n-1},
$$

where the sequence $\left\{A_{n}\right\}$ is defined as $A_{0}=1, A_{1}=a, A_{n+1}=a \cdot A_{n}+b \cdot A_{n-1}$ for all $n \geq 1$. 
It is clear that if we take $b=1$, then $X_{n}$ is the Fibonacci polynomials, see [4-7].

The main purpose of this paper is, using the elementary method and the properties of the third-order linear recurrence sequence, to unify the above results by proving the following theorem.

Theorem Let $\left\{X_{n}\right\}$ be a third-order linear recurrence sequence $X_{n+3}=a \cdot X_{n+2}+b \cdot X_{n+1}+$ $c \cdot X_{n}$ with the initial values $X_{0}=u, X_{1}=v$ and $X_{2}=w$ for all $n \geq 1$, where $a, b$ and $c$ are positive integers. For any positive integer $d \geq 2$, we define the Smarandache-Pascal derived sequence of $\left\{X_{d n+1}\right\}$ as

$$
T_{n+1}=\sum_{k=0}^{n}\left(\begin{array}{l}
n \\
k
\end{array}\right) \cdot X_{d k+1} .
$$

Then we have the recurrence formula

$$
T_{n+1}=\frac{g_{1} g_{3}-g_{1} g_{6}+g_{7}}{g_{3}-g_{6}} \cdot T_{n}+\frac{g_{3} g_{4}-g_{2} g_{6}-g_{1} g_{7}}{g_{3}-g_{6}} \cdot T_{n-1}+\frac{g_{3} g_{5}-g_{2} g_{7}}{g_{3}-g_{6}} \cdot T_{n-2},
$$

where

$$
\begin{aligned}
& g_{1}=f_{1}+f_{2}+c \cdot A_{d} f_{5}, \quad g_{2}=f_{3} A_{d+1}-f_{1} f_{2}+c \cdot A_{d} f_{6}, \\
& g_{3}=c \cdot A_{d+1}^{2}-c \cdot A_{d} f_{2}+c \cdot A_{d} f_{4}, \quad g_{4}=f_{3} A_{d+1}-f_{1} f_{2}+c \cdot A_{d}\left(f_{5}+f_{6}\right), \\
& g_{5}=c \cdot A_{d} f_{6}, \quad g_{6}=c \cdot\left(A_{d+1}-A_{d} f_{2}+A_{d} f_{4}-A_{d}\right), \quad g_{7}=c \cdot A_{d} f_{4}
\end{aligned}
$$

and

$$
\begin{aligned}
& f_{1}=b \cdot A_{d}+c \cdot A_{d-1}+1, \quad f_{2}=1+A_{d+2}, \\
& f_{3}=b \cdot A_{d-1}+c \cdot A_{d}, \quad f_{4}=1+c \cdot A_{d-1}-\frac{c \cdot A_{d}^{2}}{A_{d+1}}, \\
& f_{5}=\frac{A_{d}}{A_{d+1}}, \quad f_{6}=b \cdot A_{d-1}+c \cdot A_{d-2}-\frac{b \cdot A_{d}^{2}+c \cdot A_{d-1} A_{d}+A_{d}}{A_{d+1}},
\end{aligned}
$$

the sequence $\left\{A_{n}\right\}$ is defined by $A_{n+3}=a \cdot A_{n+2}+b \cdot A_{n+1}+c \cdot A_{n}$ with the initial values $A_{1}=0$, $A_{2}=1$ and $A_{3}=$ a for all $n \geq 1$.

From our theorem we know that if $\left\{b_{n}\right\}$ is a third-order linear recurrence sequence, then its Smarandache-Pascal derived sequence $\left\{T_{n}\right\}$ is also a third-order linear recurrence sequence.

\section{Proof of the theorem}

To complete the proof of our theorem, we need the following lemma.

Lemma Let integers $m \geq 0$ and $n \geq 3$.If the sequence $\left\{X_{n}\right\}$ satisfies the recurrence relations $X_{n+3}=a \cdot X_{n+2}+b \cdot X_{n+1}+c \cdot X_{n}, n \geq 0$, then we have the identity

$$
X_{m+n}=A_{n} \cdot X_{m+2}+\left(b \cdot A_{n-1}+c \cdot A_{n-2}\right) \cdot X_{m+1}+c \cdot A_{n-1} \cdot X_{m}
$$


where $A_{n}$ is defined by $A_{n+3}=a \cdot A_{n+2}+b \cdot A_{n+1}+c \cdot A_{n}$ with the initial values $A_{1}=0, A_{2}=1$ and $A_{3}=$ a for all $n \geq 1$.

Proof Now we prove this lemma by mathematical induction. Note that the recurrence formula $X_{m+3}=a \cdot X_{m+2}+b \cdot X_{m+1}+c \cdot X_{m}=A_{3} \cdot X_{m+2}+\left(b \cdot A_{2}+c \cdot A_{1}\right) \cdot X_{m+1}+c \cdot A_{2} \cdot X_{m}$ for all $n \geq 1$. That is, the lemma holds for $n=3$ since

$$
\begin{aligned}
X_{m+4} & =a \cdot\left(a \cdot X_{m+2}+b \cdot X_{m+1}+c \cdot X_{m}\right)+b \cdot X_{m+2}+c \cdot X_{m+1} \\
& =\left(a^{2}+b\right) \cdot X_{m+2}+(a b+c) \cdot X_{m+1}+a c \cdot X_{m} \\
& =A_{4} \cdot X_{m+2}+\left(b \cdot A_{3}+c \cdot A_{2}\right) \cdot X_{m+1}+c \cdot A_{3} \cdot X_{m} .
\end{aligned}
$$

That is, the lemma holds for $n=4$. Suppose that for all integers $2 \leq n \leq k$, we have $X_{m+n}=$ $A_{n} \cdot X_{m+2}+\left(b \cdot A_{n-1}+c \cdot A_{n-2}\right) \cdot X_{m+1}+c \cdot A_{n-1} \cdot X_{m}$. Then, for $n=k+1$, from the recurrence relations for $X_{m}$ and the inductive hypothesis, we have

$$
\begin{aligned}
X_{m+k+1}= & a \cdot X_{m+k}+b \cdot X_{m+k-1}+c \cdot X_{m+k-2} \\
= & a \cdot\left(A_{k} \cdot X_{m+2}+\left(b \cdot A_{k-1}+c \cdot A_{k-2}\right) \cdot X_{m+1}+c \cdot A_{k-1} \cdot X_{m}\right) \\
& +b \cdot\left(A_{k-1} \cdot X_{m+2}+\left(b \cdot A_{k-2}+c \cdot A_{k-3}\right) \cdot X_{m+1}+c \cdot A_{k-2} \cdot X_{m}\right) \\
& +c \cdot\left(A_{k-2} \cdot X_{m+2}+\left(b \cdot A_{k-3}+c \cdot A_{k-4}\right) \cdot X_{m+1}+c \cdot A_{k-3} \cdot X_{m}\right) \\
= & \left(a \cdot A_{k}+b \cdot A_{k-1}+c \cdot A_{k-2}\right) \cdot X_{m+2}+\left(a b \cdot A_{k-1}+\left(a c+b^{2}\right) \cdot A_{k-2}\right. \\
& \left.+2 b c \cdot A_{k-3}+c^{2} \cdot A_{k-4}\right) \cdot X_{m+1}+c\left(a \cdot A_{k-1}+b \cdot A_{k-2}+c \cdot A_{k-3}\right) \cdot X_{m} \\
= & A_{k+1} \cdot X_{m+2}+\left(a b \cdot A_{k-1}+b^{2} \cdot A_{k-2}+b c \cdot A_{k-3}+c \cdot A_{k-1}\right) \cdot X_{m+1}+c \cdot A_{k} \cdot X_{m} \\
= & A_{k+1} \cdot X_{m+2}+\left(b \cdot A_{k}+c \cdot A_{k-1}\right) \cdot X_{m+1}+c \cdot A_{k} \cdot X_{m} .
\end{aligned}
$$

That is, the lemma also holds for $n=k+1$. This completes the proof of our lemma by mathematical induction.

Now we use this lemma to complete the proof of our theorem. From the properties of the binomial coefficient $\left(\begin{array}{l}n \\ k\end{array}\right)$, we have

$$
\begin{aligned}
\left(\begin{array}{c}
n-1 \\
k
\end{array}\right)+\left(\begin{array}{l}
n-1 \\
k-1
\end{array}\right) & =\frac{(n-1) !}{k !(n-1-k) !}+\frac{(n-1) !}{(k-1) !(n-k) !} \\
& =\frac{(n-1) !}{(k-1) !(n-k-1) !}\left(\frac{1}{k}+\frac{1}{n-k}\right)=\left(\begin{array}{l}
n \\
k
\end{array}\right) .
\end{aligned}
$$

For any positive integer $d$, from the lemma we have $X_{d k+d+1}=A_{d+1} \cdot X_{d k+2}+\left(b \cdot A_{d}+c\right.$. $\left.A_{d-1}\right) \cdot X_{d k+1}+c \cdot A_{d} \cdot X_{d k}$. By the definition of $T_{n}$, we may deduce that

$$
\begin{aligned}
T_{n+1} & =\sum_{k=0}^{n}\left(\begin{array}{l}
n \\
k
\end{array}\right) \cdot X_{d k+1} \\
& =X_{1}+X_{d n+1}+\sum_{k=1}^{n-1}\left(\left(\begin{array}{c}
n-1 \\
k
\end{array}\right)+\left(\begin{array}{c}
n-1 \\
k-1
\end{array}\right)\right) \cdot X_{d k+1}
\end{aligned}
$$




$$
\begin{aligned}
= & \sum_{k=0}^{n-1}\left(\begin{array}{c}
n-1 \\
k
\end{array}\right) \cdot X_{d k+1}+\sum_{k=0}^{n-2}\left(\begin{array}{c}
n-1 \\
k
\end{array}\right) \cdot X_{d k+d+1}+X_{d n+1} \\
= & T_{n}+\sum_{k=0}^{n-1}\left(\begin{array}{c}
n-1 \\
k
\end{array}\right) \cdot X_{d k+d+1} \\
= & T_{n}+\sum_{k=0}^{n-1}\left(\begin{array}{c}
n-1 \\
k
\end{array}\right) \cdot\left(A_{d+1} \cdot X_{d k+2}+\left(b \cdot A_{d}+c \cdot A_{d-1}\right) \cdot X_{d k+1}+c \cdot A_{d} \cdot X_{d k}\right) \\
= & \left(b \cdot A_{d}+c \cdot A_{d-1}+1\right) \cdot T_{n}+A_{d+1} \sum_{k=0}^{n-1}\left(\begin{array}{c}
n-1 \\
k
\end{array}\right) \cdot X_{d k+2} \\
& +c \cdot A_{d} \sum_{k=0}^{n-1}\left(\begin{array}{c}
n-1 \\
k
\end{array}\right) \cdot X_{d k} .
\end{aligned}
$$

For convenience, we let $f_{1}\left(A_{k}\right)=b \cdot A_{d}+c \cdot A_{d-1}+1$ (briefly $f_{1}$ ), then the above identity implies that

$$
T_{n+1}=f_{1} \cdot T_{n}+A_{d+1} \sum_{k=0}^{n-1}\left(\begin{array}{c}
n-1 \\
k
\end{array}\right) \cdot X_{d k+2}+c \cdot A_{d} \sum_{k=0}^{n-1}\left(\begin{array}{c}
n-1 \\
k
\end{array}\right) \cdot X_{d k}
$$

From this identity, we can also deduce

$$
T_{n}=f_{1} \cdot T_{n-1}+A_{d+1} \sum_{k=0}^{n-2}\left(\begin{array}{c}
n-2 \\
k
\end{array}\right) \cdot X_{d k+2}+c \cdot A_{d} \sum_{k=0}^{n-2}\left(\begin{array}{c}
n-2 \\
k
\end{array}\right) \cdot X_{d k}
$$

and

$$
T_{n-1}=f_{1} \cdot T_{n-2}+A_{d+1} \sum_{k=0}^{n-3}\left(\begin{array}{c}
n-3 \\
k
\end{array}\right) \cdot X_{d k+2}+c \cdot A_{d} \sum_{k=0}^{n-3}\left(\begin{array}{c}
n-3 \\
k
\end{array}\right) \cdot X_{d k} .
$$

They are equivalent to

$$
\sum_{k=0}^{n-2}\left(\begin{array}{c}
n-2 \\
k
\end{array}\right) \cdot X_{d k+2}=\frac{1}{A_{d+1}}\left(T_{n}-f_{1} \cdot T_{n-1}-c \cdot A_{d} \sum_{k=0}^{n-2}\left(\begin{array}{c}
n-2 \\
k
\end{array}\right) \cdot X_{d k}\right)
$$

and

$$
\sum_{k=0}^{n-3}\left(\begin{array}{c}
n-3 \\
k
\end{array}\right) \cdot X_{d k+2}=\frac{1}{A_{d+1}}\left(T_{n-1}-f_{1} \cdot T_{n-2}-c \cdot A_{d} \sum_{k=0}^{n-3}\left(\begin{array}{c}
n-3 \\
k
\end{array}\right) \cdot X_{d k}\right)
$$

On the other hand, from the lemma we also deduce $X_{d k+d+2}=A_{d+2} \cdot X_{d k+2}+\left(b \cdot A_{d+1}+\right.$ $\left.c \cdot A_{d}\right) \cdot X_{d k+1}+c \cdot A_{d+1} \cdot X_{d k}$. Then we have

$$
\begin{aligned}
& \sum_{k=0}^{n-1}\left(\begin{array}{c}
n-1 \\
k
\end{array}\right) \cdot X_{d k+2} \\
& \quad=X_{2}+X_{d n-d+2}+\sum_{k=1}^{n-2}\left(\begin{array}{c}
n-1 \\
k
\end{array}\right) \cdot X_{d k+2}
\end{aligned}
$$




$$
\begin{aligned}
= & X_{2}+X_{d n-d+2}+\sum_{k=1}^{n-2}\left(\left(\begin{array}{c}
n-2 \\
k
\end{array}\right)+\left(\begin{array}{c}
n-2 \\
k-1
\end{array}\right)\right) \cdot X_{d k+2} \\
= & \sum_{k=0}^{n-2}\left(\begin{array}{c}
n-2 \\
k
\end{array}\right) \cdot X_{d k+d+2}+\sum_{k=0}^{n-2}\left(\begin{array}{c}
n-2 \\
k
\end{array}\right) \cdot X_{d k+2} \\
= & \sum_{k=0}^{n-2}\left(\begin{array}{c}
n-2 \\
k
\end{array}\right) \cdot\left(A_{d+2} \cdot X_{d k+2}+\left(b \cdot A_{d+1}+c \cdot A_{d}\right) \cdot X_{d k+1}+c \cdot A_{d+1} \cdot X_{d k}\right) \\
& +\sum_{k=0}^{n-2}\left(\begin{array}{c}
n-2 \\
k
\end{array}\right) \cdot X_{d k+2} \\
= & \left(1+A_{d+2}\right) \cdot \sum_{k=0}^{n-2}\left(\begin{array}{c}
n-2 \\
k
\end{array}\right) \cdot X_{d k+2}+c \cdot A_{d+1} \sum_{k=0}^{n-2}\left(\begin{array}{c}
n-2 \\
k
\end{array}\right) \cdot X_{d k} \\
& +\left(b \cdot A_{d+1}+c \cdot A_{d}\right) \cdot T_{n-1} .
\end{aligned}
$$

Similarly, applying formula (1) and identity (3), we have

$$
\begin{aligned}
& \sum_{k=0}^{n-1}\left(\begin{array}{c}
n-1 \\
k
\end{array}\right) \cdot X_{d k} \\
& =X_{0}+X_{d n-d}+\sum_{k=1}^{n-2}\left(\begin{array}{c}
n-1 \\
k
\end{array}\right) \cdot X_{d k} \\
& =X_{0}+X_{d n-d}+\sum_{k=1}^{n-2}\left(\left(\begin{array}{c}
n-2 \\
k
\end{array}\right)+\left(\begin{array}{c}
n-2 \\
k-1
\end{array}\right)\right) \cdot X_{d k} \\
& =\sum_{k=0}^{n-2}\left(\begin{array}{c}
n-2 \\
k
\end{array}\right) \cdot\left(A_{d} \cdot X_{d k+2}+\left(b \cdot A_{d-1}+c \cdot A_{d-2}\right) \cdot X_{d k+1}+c \cdot A_{d-1} \cdot X_{d k}\right) \\
& +\sum_{k=0}^{n-2}\left(\begin{array}{c}
n-2 \\
k
\end{array}\right) \cdot X_{d k} \\
& =A_{d} \cdot \sum_{k=0}^{n-2}\left(\begin{array}{c}
n-2 \\
k
\end{array}\right) \cdot X_{d k+2}+\left(1+c \cdot A_{d-1}\right) \sum_{k=0}^{n-2}\left(\begin{array}{c}
n-2 \\
k
\end{array}\right) \cdot X_{d k} \\
& +\left(b \cdot A_{d-1}+c \cdot A_{d-2}\right) \cdot T_{n-1} \\
& =\frac{A_{d}}{A_{d+1}}\left(T_{n}-\left(b \cdot A_{d}+c \cdot A_{d-1}+1\right) \cdot T_{n-1}-c \cdot A_{d} \sum_{k=0}^{n-2}\left(\begin{array}{c}
n-2 \\
k
\end{array}\right) \cdot X_{d k}\right) \\
& +\left(1+c \cdot A_{d-1}\right) \sum_{k=0}^{n-2}\left(\begin{array}{c}
n-2 \\
k
\end{array}\right) \cdot X_{d k} \\
& +\left(b \cdot A_{d-1}+c \cdot A_{d-2}\right) \cdot T_{n-1} \\
& =\frac{A_{d}}{A_{d+1}} \cdot T_{n}+\left(b \cdot A_{d-1}+c \cdot A_{d-2}-\frac{b \cdot A_{d}^{2}+c \cdot A_{d-1} A_{d}+A_{d}}{A_{d+1}}\right) \cdot T_{n-1} \\
& +\left(1+c \cdot A_{d-1}-\frac{c \cdot A_{d}^{2}}{A_{d+1}}\right) \sum_{k=0}^{n-2}\left(\begin{array}{c}
n-2 \\
k
\end{array}\right) \cdot X_{d k} .
\end{aligned}
$$


For convenience, we let

$$
\begin{aligned}
& f_{2}=1+A_{d+2}, \quad f_{3}=b \cdot A_{d+1}+c \cdot A_{d}, \quad f_{4}=1+c \cdot A_{d-1}-\frac{c \cdot A_{d}^{2}}{A_{d+1}}, \\
& f_{5}=\frac{A_{d}}{A_{d+1}}, \quad f_{6}=b \cdot A_{d-1}+c \cdot A_{d-2}-\frac{b \cdot A_{d}^{2}+c \cdot A_{d-1} A_{d}+A_{d}}{A_{d+1}}
\end{aligned}
$$

then identities (5) and (6) imply that

$$
\begin{aligned}
& \sum_{k=0}^{n-1}\left(\begin{array}{c}
n-1 \\
k
\end{array}\right) \cdot X_{d k+2} \\
& \quad=f_{2} \cdot \sum_{k=0}^{n-2}\left(\begin{array}{c}
n-2 \\
k
\end{array}\right) \cdot X_{d k+2}+c \cdot A_{d+1} \sum_{k=0}^{n-2}\left(\begin{array}{c}
n-2 \\
k
\end{array}\right) \cdot X_{d k}+f_{3} \cdot T_{n-1}, \\
& \sum_{k=0}^{n-1}\left(\begin{array}{c}
n-1 \\
k
\end{array}\right) \cdot X_{d k}=f_{4} \sum_{k=0}^{n-2}\left(\begin{array}{c}
n-2 \\
k
\end{array}\right) \cdot X_{d k}+f_{5} T_{n}+f_{6} T_{n-1} .
\end{aligned}
$$

Combining (2), (3), (7) and (8), we deduce

$$
\begin{aligned}
T_{n+1}= & \left(f_{1}+f_{2}+c \cdot A_{d} f_{5}\right) \cdot T_{n}+\left(f_{3} A_{d+1}-f_{1} f_{2}+c \cdot A_{d} f_{6}\right) \cdot T_{n-1} \\
& +\left(c \cdot A_{d+1}^{2}-c \cdot A_{d} f_{2}+c \cdot A_{d} f_{4}\right) \cdot \sum_{k=0}^{n-2}\left(\begin{array}{c}
n-2 \\
k
\end{array}\right) \cdot X_{d k} .
\end{aligned}
$$

Applying formula (1), we deduce

$$
\left(\begin{array}{l}
n \\
k
\end{array}\right)=\left(\begin{array}{c}
n-1 \\
k
\end{array}\right)+\left(\begin{array}{l}
n-1 \\
k-1
\end{array}\right)=\left(\begin{array}{c}
n-2 \\
k
\end{array}\right)+\left(\begin{array}{l}
n-2 \\
k-1
\end{array}\right)+\left(\begin{array}{l}
n-1 \\
k-1
\end{array}\right) .
$$

From this and identities (3) and (4), note that $X_{d k+d}=A_{d} \cdot X_{d k+2}+\left(b \cdot A_{d-1}+c \cdot A_{d-2}\right)$. $X_{d k+1}+c \cdot A_{d-1} \cdot X_{d k}$, we have

$$
\begin{aligned}
\sum_{k=0}^{n-1}\left(\begin{array}{c}
n-1 \\
k
\end{array}\right) \cdot X_{d k} & \\
= & X_{0}+X_{d n-d}+\left(\begin{array}{c}
n-1 \\
n-2
\end{array}\right) \cdot X_{d n-2 d}+\sum_{k=1}^{n-3}\left(\begin{array}{c}
n-1 \\
k
\end{array}\right) \cdot X_{d k} \\
= & X_{0}+X_{d n-d}+(n-1) \cdot X_{d n-2 d}+\sum_{k=1}^{n-3}\left(\left(\begin{array}{c}
n-3 \\
k
\end{array}\right)+\left(\begin{array}{c}
n-3 \\
k-1
\end{array}\right)+\left(\begin{array}{c}
n-2 \\
k-1
\end{array}\right)\right) \cdot X_{d k} \\
= & X_{0}+X_{d n-d}+(n-1) \cdot X_{d n-2 d}+\sum_{k=0}^{n-3}\left(\begin{array}{c}
n-3 \\
k
\end{array}\right) \cdot X_{d k}-X_{0} \\
& +\sum_{k=0}^{n-3}\left(\begin{array}{c}
n-3 \\
k
\end{array}\right) \cdot X_{d k+d}-X_{d n-2 d}+\sum_{k=0}^{n-2}\left(\begin{array}{c}
n-2 \\
k
\end{array}\right) \cdot X_{d k+d}-(n-2) \cdot X_{d n-2 d}-X_{d n-d} \\
= & \sum_{k=0}^{n-3}\left(\begin{array}{c}
n-3 \\
k
\end{array}\right) \cdot X_{d k}+\sum_{k=0}^{n-3}\left(\begin{array}{c}
n-3 \\
k
\end{array}\right) \cdot X_{d k+d}+\sum_{k=0}^{n-2}\left(\begin{array}{c}
n-2 \\
k
\end{array}\right) \cdot X_{d k+d}
\end{aligned}
$$




$$
\begin{aligned}
= & A_{d} \sum_{k=0}^{n-2}\left(\begin{array}{c}
n-2 \\
k
\end{array}\right) \cdot X_{d k+2}+A_{d} \sum_{k=0}^{n-3}\left(\begin{array}{c}
n-3 \\
k
\end{array}\right) \cdot X_{d k+2}+c \cdot A_{d-1} \sum_{k=0}^{n-2}\left(\begin{array}{c}
n-2 \\
k
\end{array}\right) \cdot X_{d k} \\
& +\left(c \cdot A_{d-1}+1\right) \sum_{k=0}^{n-3}\left(\begin{array}{c}
n-3 \\
k
\end{array}\right) \cdot X_{d k}+\left(b \cdot A_{d-1}+c \cdot A_{d-2}\right) \cdot\left(T_{n-1}+T_{n-2}\right) \\
= & \frac{A_{d}}{A_{d+1}} T_{n}+\left(b \cdot A_{d-1}+c \cdot A_{d-2}-\frac{b \cdot A_{d}^{2}+c \cdot A_{d-1} A_{d}}{A_{d+1}}\right) \cdot T_{n-1} \\
& +\left(b \cdot A_{d-1}+c \cdot A_{d-2}-\frac{b \cdot A_{d}^{2}+c \cdot A_{d-1} A_{d}+A_{d}}{A_{d+1}}\right) \cdot T_{n-2} \\
& +\left(c \cdot A_{d-1}-\frac{c \cdot A_{d}^{2}}{A_{d+1}}\right) \sum_{k=0}^{n-2}\left(\begin{array}{c}
n-2 \\
k
\end{array}\right) \cdot X_{d k} \\
& +\left(c \cdot A_{d-1}+1-\frac{c \cdot A_{d}^{2}}{A_{d+1}}\right) \sum_{k=0}^{n-3}\left(\begin{array}{c}
n-3 \\
k
\end{array}\right) \cdot X_{d k} \\
= & f_{5} T_{n}+\left(f_{5}+f_{6}\right) T_{n-1}+f_{6} T_{n-2} \\
& +\left(f_{4}-1\right) \sum_{k=0}^{n-2}\left(\begin{array}{c}
n-2 \\
k
\end{array}\right) \cdot X_{d k}+f_{4} \sum_{k=0}^{n-3}\left(\begin{array}{c}
n-3 \\
k
\end{array}\right) \cdot X_{d k} .
\end{aligned}
$$

Combining (2), (3), (7) and (11), we deduce

$$
\begin{aligned}
T_{n+1}= & \left(f_{1}+f_{2}+c \cdot A_{d} f_{5}\right) \cdot T_{n}+\left(f_{3} A_{d+1}-f_{1} f_{2}+c \cdot A_{d}\left(f_{5}+f_{6}\right)\right) \cdot T_{n-1}+c \cdot A_{d} f_{6} \cdot T_{n-2} \\
& +c\left(A_{d+1}-A_{d} f_{2}+A_{d} f_{4}-A_{d}\right) \cdot \sum_{k=0}^{n-2}\left(\begin{array}{c}
n-2 \\
k
\end{array}\right) \cdot X_{d k} \\
& +c \cdot A_{d} f_{4} \cdot \sum_{k=0}^{n-3}\left(\begin{array}{c}
n-3 \\
k
\end{array}\right) \cdot X_{d k} .
\end{aligned}
$$

From identity (9) we can also deduce

$$
\begin{aligned}
T_{n}= & \left(f_{1}+f_{2}+c \cdot A_{d} f_{5}\right) \cdot T_{n-1}+\left(f_{3} A_{d+1}-f_{1} f_{2}+c \cdot A_{d} f_{6}\right) \cdot T_{n-2} \\
& +\left(c \cdot A_{d+1}^{2}-c \cdot A_{d} f_{2}+c \cdot A_{d} f_{4}\right) \cdot \sum_{k=0}^{n-3}\left(\begin{array}{c}
n-3 \\
k
\end{array}\right) \cdot X_{d k} .
\end{aligned}
$$

For convenience, we let

$$
\begin{aligned}
& g_{1}=f_{1}+f_{2}+c \cdot A_{d} f_{5}, \quad g_{2}=f_{3} A_{d+1}-f_{1} f_{2}+c \cdot A_{d} f_{6}, \\
& g_{3}=c \cdot A_{d+1}^{2}-c \cdot A_{d} f_{2}+c \cdot A_{d} f_{4}, \quad g_{4}=f_{3} A_{d+1}-f_{1} f_{2}+c \cdot A_{d}\left(f_{5}+f_{6}\right), \\
& g_{5}=c \cdot A_{d} f_{6}, \quad g_{6}=c \cdot\left(A_{d+1}-A_{d} f_{2}+A_{d} f_{4}-A_{d}\right), \quad g_{7}=c \cdot A_{d} f_{4} .
\end{aligned}
$$

Inserting (9) and (13) into (12), we deduce

$$
T_{n+1}=\frac{g_{1} g_{3}-g_{1} g_{6}+g_{7}}{g_{3}-g_{6}} \cdot T_{n}+\frac{g_{3} g_{4}-g_{2} g_{6}-g_{1} g_{7}}{g_{3}-g_{6}} \cdot T_{n-1}+\frac{g_{3} g_{5}-g_{2} g_{7}}{g_{3}-g_{6}} \cdot T_{n-2} .
$$

This completes the proof of our theorem. 
Remark In fact, using the above formulas, we can also obtain the recurrence formula of the Smarandache-Pascal derived sequence $\left\{T_{n}\right\}$ of $\left\{u_{n}\right\}$, where $\left\{u_{n}\right\}$ denotes the $m$ th-order linear recursive sequences as follows:

$$
u_{n}=a_{1} u_{n-1}+a_{2} u_{n-2}+\cdots+a_{m-1} u_{n-m+1}+a_{m} u_{n-m},
$$

with initial values $u_{i} \in \mathbb{N}$ for $n>m$ and $0 \leq i<m$.

\section{Competing interests}

The authors declare that they have no competing interests.

\section{Authors' contributions}

ZW obtained the theorems and completed the proof. $\mathrm{J}$ and $\mathrm{HZ}$ corrected and improved the final version. All authors read and approved the final manuscript.

\section{Author details}

'Department of Mathematics, Northwest University, Xi'an, Shaanxi, P.R. China. ${ }^{2}$ College of Science, Xi'an University of Technology, Xi'an, Shaanxi, P.R. China.

\section{Acknowledgements}

The authors express their gratitude to the referee for very helpful and detailed comments. This work is supported by the N.S.F. $(11071194,11001218)$ of P.R. China and the G.I.C.F. (YZZ12062) of NWU.

Received: 8 August 2013 Accepted: 29 August 2013 Published: 07 Nov 2013

\section{References}

1. Smarandache, F: Only Problems, Not Solutions. Xiquan Publishing House, Chicago (1993)

2. Murthy, A, Ashbacher, C: Generalized Partitions and New Ideas on Number Theory and Smarandache Sequences. Hexis, Phoenix (2005)

3. Li, X, Han, D: On the Smarandache-Pascal derived sequences and some of their conjectures. Adv. Differ. Equ. 2013, 240 (2013)

4. $\mathrm{Ma}, \mathrm{R}$, Zhang, W: Several identities involving the Fibonacci numbers and Lucas numbers. Fibonacci Q. 45, 164-170 (2007)

5. $Y i, Y, Z$ hang, $W$ : Some identities involving the Fibonacci polynomials. Fibonacci Q. 40, 314-318 (2002)

6. Wang, T, Zhang, W: Some identities involving Fibonacci, Lucas polynomials and their applications. Bull. Math. Soc. Sci. Math. Roum. 55, 95-103 (2012)

7. Riordan, J: Combinatorial Identities. Wiley, New York (1968)

10.1186/1687-1847-2013-284

Cite this article as: Wu et al.: On the Smarandache-Pascal derived sequences of generalized Tribonacci numbers Advances in Difference Equations 2013, 2013:284

\section{Submit your manuscript to a SpringerOpen ${ }^{\circ}$ journal and benefit from:}

- Convenient online submission

Rigorous peer review

- Immediate publication on acceptance

- Open access: articles freely available online

- High visibility within the field

- Retaining the copyright to your article 